\title{
Evidence-based management of central cord syndrome
}

\author{
Nader S. Dahdaleh, M.D., Cort D. Lawton, B.S., Tarek Y. El Ahmadieh, M.D., \\ Alexander T. Nixon, M.S., Najib E. El Tecle, M.D., Sanders OH, B.S., \\ Richard G. Fessler, M.D., Ph.D., and Zachary A. Smith, M.D.
}

Department of Neurological Surgery, Northwestern University, Chicago, Illinois

\begin{abstract}
Object. Evidence-based medicine is used to examine the current treatment options, timing of surgical intervention, and prognostic factors in the management of patients with traumatic central cord syndrome (TCCS).

Methods. A computerized literature search of the National Library of Medicine database, Cochrane database, and Google Scholar was performed for published material between January 1966 and February 2013 using key words and Medical Subject Headings. Abstracts were reviewed and selected, with the articles segregated into 3 main categories: surgical versus conservative management, timing of surgery, and prognostic factors. Evidentiary tables were then assembled, summarizing data and quality of evidence (Classes I-III) for papers included in this review.

Results. The authors compiled 3 evidentiary tables summarizing 16 studies, all of which were retrospective in design. Regarding surgical intervention versus conservative management, there was Class III evidence to support the superiority of surgery for patients presenting with TCCS. In regards to timing of surgery, most Class III evidence demonstrated no difference in early versus late surgical management. Most Class III studies agreed that older age, especially age greater than 60-70 years, correlated with worse outcomes.

Conclusions. No Class I or Class II evidence was available to determine the efficacy of surgery, timing of surgical intervention, or prognostic factors in patients managed for TCCS. Hence, there is a need to perform wellcontrolled prospective studies and randomized controlled clinical trials to further investigate the optimal management (surgical vs conservative) and timing of surgical intervention in patients suffering from TCCS.

(http://thejns.org/doi/abs/10.3171/2013.3.FOCUS13101)
\end{abstract}

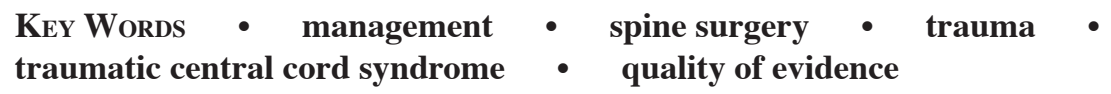

A CUTE TCCS was first described by Schneider et al. in 1954 as a "syndrome of acute central cervical spinal cord injury characterized by disproportionately more impairment of the upper than the lower extremities, bladder dysfunction, usually urinary retention, and varying degrees of sensory loss below the level of the lesion." 22 Maroon et al. described burning, paresthesias, and dysesthesias in the hands as some patients' only complaint. ${ }^{17}$ It is now recognized that this syndrome presents as a spectrum from weakness limited to the upper extremity with sensory preservation, to complete quadriparesis with sacral sparing..$^{20}$ Causes of TCCS are variable and may result from unstable cervical fractures and fracture dislocations, acute disc herniations, or following hyperextension injury in the setting of preexisting cervical

\footnotetext{
Abbreviations used in this paper: ASIA = American Spinal Injury Association; FIM = Functional Independence Measure; JOA = Japanese Orthopaedic Association; MBI = modified Barthel Index; OPLL = ossification of the posterior longitudinal ligament; SF-36 = 36-Item Short Form Health Survey; TCCS = traumatic central cord syndrome.
}

spondylotic changes (including OPLL) in the absence of bone fractures. ${ }^{9,12,15,23}$

To date, the exact pathophysiology remains controversial. The mechanism proposed by Schneider et al. suggested that central spinal cord compression following hyperextension injury resulted in injury to the central spinal cord tracts. ${ }^{22}$ More recently, histopathological examinations and MR correlations have suggested that the location of the injury may be in the lateral corticospinal tracts, resulting in a disproportionate loss of upper extremity (namely the hand) than lower extremity function. ${ }^{14,21,24}$

Modern radiological evaluation and classification schemes are often employed to aid in assigning spinal stability in cases of cervical fractures. Patients suffering from TCCS secondary to acute disc herniations, fractures, and/or instability are usually managed surgically. ${ }^{2,9,12}$ The current controversy lies in managing patients suffering from TCCS secondary to a hyperextension injury in the setting of preexisting cervical stenotic changes without fractures or instability, whether to intervene surgically or medically, and on the timing of surgical intervention. Evidence-based management articles for 
TCCS have been lacking in the literature. We attempted to systematically review current evidence associated with this type of injury.

\section{Methods}

A computerized literature search of the National Library of Medicine database, Cochrane database, and Google Scholar was performed for material published between January 1966 and February 2013 using key words and Medical Subject Headings; key words included: central cord syndrome, traumatic central cord syndrome, acute traumatic central cord syndrome, and central cord syndrome surgery. The search yielded 1675 citations. A total of 162 citations pertained to management of central cord syndrome, which was narrowed down to 77 citations after accounting for redundancy. We then selected for English citations and reviewed all abstracts generated in the search.

Among the citations reviewed, we identified 16 articles that addressed conservative versus surgical management, timing of surgery, and/or prognostic determinants for patients treated for TCCS. All 16 citations were retrospective studies (Tables 1-3). The articles were separated into 3 categories: outcomes of surgery versus conservative management, timing of surgery, and analysis of prognostic factors. Articles were classified according to the level of evidence (I-III). ${ }^{18}$

\section{Results}

\section{Surgery Versus Conservative Management}

Four articles, all of which were retrospective studies, compared surgery to conservative treatment for TCCS (Table 1). In 1984, Bose et al. retrospectively studied 28 patients treated for central cord syndrome. ${ }^{5}$ Fourteen patients underwent medical management consisting of immobilization, mannitol, dexamethasone, and sodium bicarbonate. The other group was treated with similar medical care and underwent additional surgery; indications for surgery included failure of continued improvement and/or presence of instability. The baseline ASIA score for both groups was similar. The degree of improvement was significantly greater in the surgically treated group. There were substantial differences in baseline characteristics between the study groups, mainly the degree of cervical instability.

In 1997, Chen et al. retrospectively reviewed 114 patients with TCCS. ${ }^{8}$ Twenty-eight patients received surgical intervention, while the remaining patients were treated conservatively. The cohort included patients with heterogeneous pathology. Patients were segregated into 4 groups based on age: Group A, 4-19 years old; Group B, 20-40 years old; Group C, 41-60 years old; and Group D, 61-75 years old. The best outcomes were observed in patients of younger age and those treated with early surgical intervention.

In 1998, Chen et al. retrospectively compared 16 patients treated surgically with 21 patients treated conservatively for acute incomplete cervical spinal cord injury. ${ }^{7}$ All patients had incomplete spinal cord injury due to minor

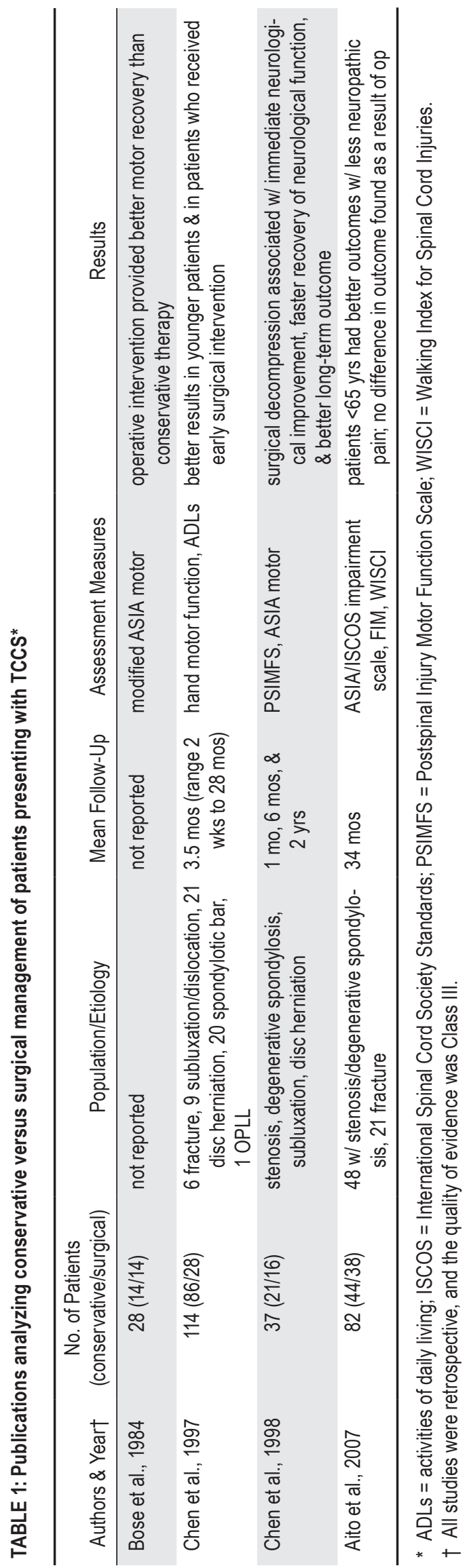

Neurosurg Focus / Volume 35 / July 2013 
Central cord syndrome

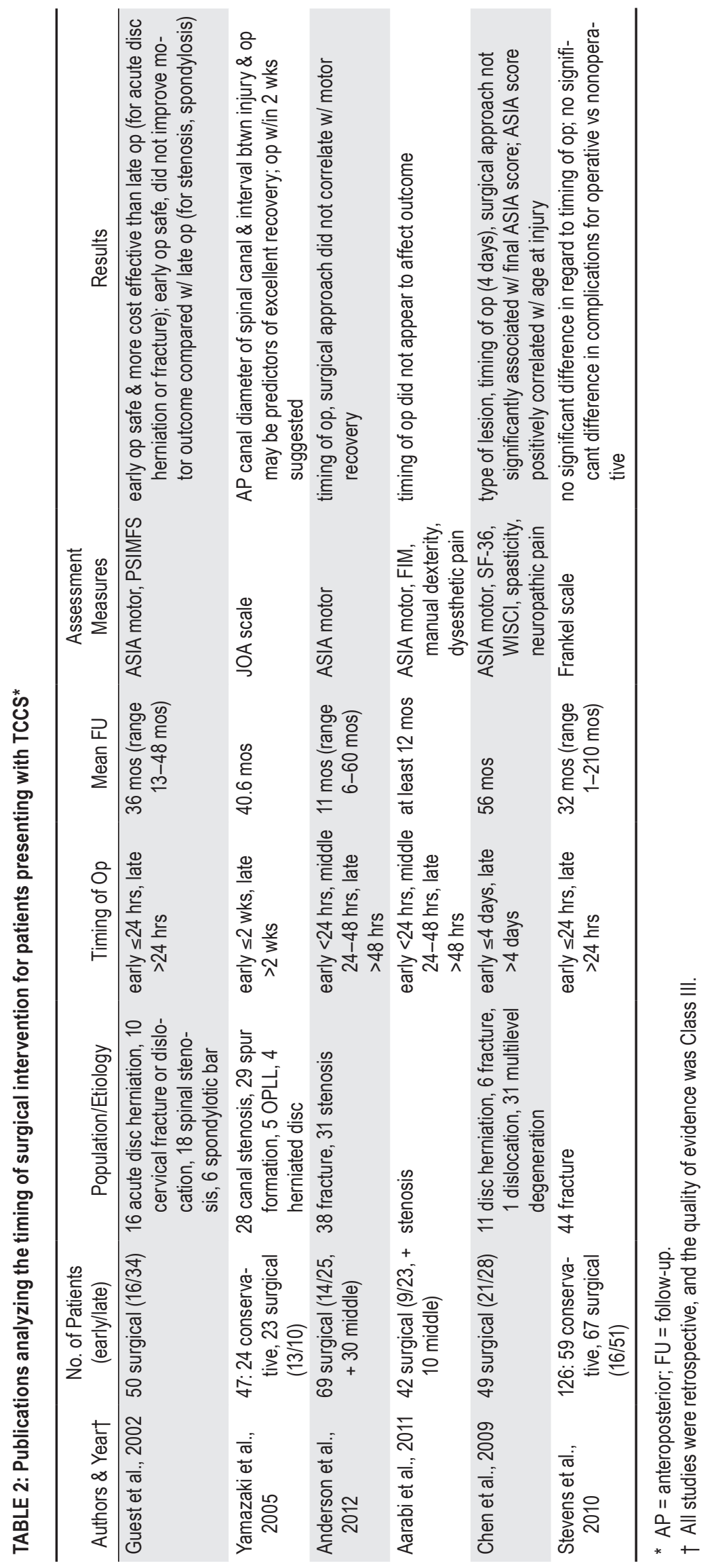




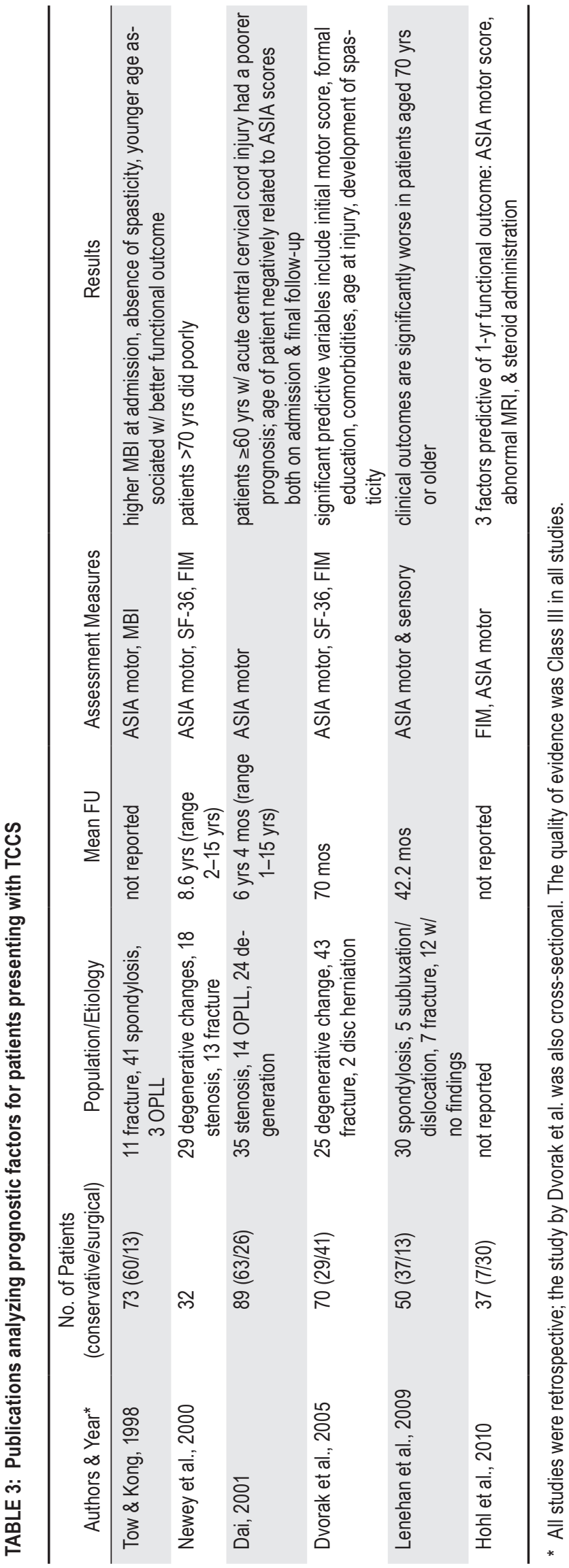

neck injury with preexisting spondylosis. Comparing the improvement of the 2 groups at defined intervals, there were statistically significant differences between the surgical and nonsurgical patients at 1-month and 6-month follow-ups. Of those patients treated surgically, $81.2 \%$ improved within 2 days, while $62 \%$ of patients treated nonoperatively had recovered to at least motor muscle Grade 3 at the 2-year follow-up; however, their recovery was slower than that of the surgical group.

In 2007, Aito et al. retrospectively compared 38 patients treated surgically for TCCS with 44 patients treated conservatively. ${ }^{3}$ The outcomes between both groups were similar in regard to ASIA motor scores. The baseline characteristics between both groups were different as the surgically treated group had skeletal and discoligamentous injuries, while the conservatively treated group suffered hyperextension injuries.

\section{Timing of Surgery}

Six articles were identified that primarily addressed timing of surgery for TCCS, all of which were retrospective studies (Table 2). In 2002, Guest et al. retrospectively reviewed 50 patients presenting with TCCS. ${ }^{12}$ Comparison of the clinical outcomes between patients undergoing early surgery (within 24 hours) or late surgery ( $>24$ hours) was conducted. The outcome measure used was the ASIA motor scale. Twenty-six patients with traumatic herniated discs and cervical fractures or dislocation were grouped together. Of those 26 patients, 10 underwent early surgery, and 16 underwent late surgery. In a second group, 24 patients with cervical spondylosis or spinal stenosis were included together; of those, 6 underwent early surgery and 18 underwent late surgery. The mean follow-up was 36 months. On average, all patients improved postoperatively. In the disc herniation and fracture group, patients undergoing early surgery improved to a greater extent than those undergoing later surgery. There was no difference in outcome when comparing early versus late surgery in patients with underlying spondylosis and stenosis. Patients older than 60 years had worse outcomes than younger patients.

In 2005, Yamazaki et al. retrospectively analyzed the clinical and radiographic data on 47 patients who were treated for TCCS due to spondylosis and disc herniations. ${ }^{26}$ The outcome measure used was the JOA score. Twenty-four patients were treated conservatively. Twentythree patients were treated surgically and divided into 2 groups: early surgery ( $\leq 2$ weeks) and late surgery $(>2$ weeks). All groups improved postoperatively. The interval between injury and surgery was predictive of excellent recovery. Within the surgically treated group, timely surgery was found to improve outcome. In the conservatively treated group, therapy did not improve patients with low JOA scores.

In 2012, Anderson et al. retrospectively reviewed 69 patients who underwent surgical treatment for TCCS. ${ }^{4}$ Fifty-five percent of these patients suffered fractures and the rest suffered TCCS in the setting of preexisting stenosis. Fourteen patients underwent surgery within 24 hours, 30 between 24 and 48 hours, and 25 more than 48 hours after injury. The average time to surgical intervention was 2.9 days. All patients received methylprednisolone 
according to National Acute Spinal Cord Injury Study (NASCIS II) criteria, and the mean arterial pressure was maintained above $85 \mathrm{~mm} \mathrm{Hg}$. Overall, patients' ASIA scores improved from 63.2 to 89.9 at final follow-up. Neither the timing of surgery nor the approach affected outcome.

In a retrospective study examining patients with TCCS due to preexisting stenosis, Aarabi et al. examined 42 patients, all of whom underwent surgery. ${ }^{1}$ The mean admission ASIA score improved significantly during the last follow-up. Timing from injury to surgery was within 24 hours in 9 patients, 24-48 hours in 10 patients, and more than 48 hours in 23 patients. American Spinal Injury Association motor score, FIM, manual dexterity, and dysesthetic pain at follow-up correlated with admission ASIA motor score, maximum canal compromise, midsagittal diameter, length of parenchymal damage on MRI, and age. In this study, timing of surgery did not appear to affect outcome.

In 2009, Chen et al. retrospectively reviewed 49 patients with TCCS. ${ }^{6}$ Outcome measures included ASIA scores and the SF-36. Twenty-seven of these patients had TCCS with associated spondylosis, while the rest were found to have disc herniations and/or fracture/dislocation. The average admission ASIA score improved at 6 months and plateaued during the last follow-up. There was no significant difference in outcome between patients who underwent surgery within 4 days of injury or after 4 days or injury; age was the only factor that influenced outcome.

In 2010, Stevens et al. retrospectively reviewed 126 patients with TCCS; ${ }^{24}$ of these patients, 44 presented with cervical fractures. Sixty-seven patients received operative treatment, while 59 were managed nonoperatively. Among those managed operatively, 16 received surgery within 24 hours after the time of injury, and 34 patients received surgery after 24 hours from injury during their first hospitalization (mean 6.4 days), while 17 patients received surgery on a second hospitalization (mean 137 days). Surgically treated patients had better Frankel scores at follow-up compared with the conservatively treated group. No statistically significant difference was observed in outcome for patients treated surgically based on the timing of intervention.

\section{Prognostic Factors}

Six articles were identified that primarily addressed prognostic factors other than timing of surgery affecting outcomes in patients with TCCS (Table 3). In 1998, Tow and Kong retrospectively reviewed 73 patients treated with TCCS, ${ }^{25} 11$ of whom had cervical fractures. Thirteen patients underwent surgical intervention. Significant improvement in ASIA scores and the MBI was noted following rehabilitation. Multiple regression analysis showed that higher admission MBI scores, absence of spasticity, and younger age correlated with better outcomes.

In 2000 , Newey et al. retrospectively studied 32 patients with central cord syndrome treated conservatively. ${ }^{19}$ Patients were segregated into 3 groups: age younger than 50 years, between 50 and 70 years, and older than 70 years. The mean follow-up was 8.6 years. The ASIA scores improved following discharge, and patients older than 70 years had worse outcomes.
Dai in 2001 retrospectively reviewed 89 patients with acute central cervical cord injury. ${ }^{10}$ There were 51 patients who suffered hyperextension injuries. Twenty-six patients were treated operatively and 63 nonoperatively. The average follow-up was 6 years 4 months. Patients were segregated into 2 groups: age younger than 60 years or age 60 years or older. Linear regression analysis was used to correlate age with ASIA scores. The study showed that age negatively correlated with ASIA scores both on admission and at follow-up. Patients 60 years of age or older had a worse prognosis, although a mild neurological improvement was noted.

In the study conducted by Aito et al., older age significantly correlated with poorer neurological improvement in groups treated both conservatively and surgically. ${ }^{3}$ In 2005, Dvorak et al. retrospectively analyzed 70 patients who were managed with TCCS: ${ }^{11}$ follow-up was at least 2 years. Cervical fractures were treated operatively. Patients with spondylosis underwent bracing, and surgery was only undertaken if the patient's neurological examination results plateaued or deteriorated. Outcome measures included ASIA scores, FIM, and SF-36. The average admission ASIA motor score significantly improved postoperatively. Potential confounders were analyzed using regression modeling. Significant predictive variables included initial motor score, formal education, comorbidities, age at injury, and development of spasticity. The FIM motor score was also higher in patients treated surgically.

In 2009, Lenehan et al. retrospectively analyzed 50 patients treated for TCCS. ${ }^{16}$ Thirteen patients were treated surgically. The patients were separated into 3 groups: younger than 50 years, between 50 and 70 years, and older than 70 years. Average follow-up was 42.2 months. Clinical outcome, including sphincter disturbance, was worse in patients older than 70 years.

In 2010, Hohl et al. retrospectively followed 37 patients treated for TCCS. ${ }^{13}$ Among these patients, 7 were treated conservatively, and 30 were treated surgically. Factors influencing 1-year FIM were analyzed. American Spinal Injury Association score at the time of presentation, ambulatory status after injury, administration of steroids, MRI evidence of abnormal signal intensity, and motor FIM at time of rehabilitation correlated with outcome, but age did not.

\section{Discussion}

The causes of TCCS are variable. While most authors agree to surgically manage acute disc herniations and unstable spine fractures in an expedited fashion, there remains controversy regarding managing patients with classical central cord syndrome that occurs as a result of hyperextension injury in the setting of spondylosis without evidence of fracture or instability. There is debate not only in whether to manage these patients surgically, but also in determining the optimal timing of surgical intervention.

We conducted a literature review and created evidentiary tables representing current evidence regarding the management of TCCS. No studies were classified as Class I or II evidence. All 16 articles were retrospective in 
design and therefore were Class III studies. Most studies had heterogeneous patient populations with TCCS that resulted from acute disc herniations, unstable spine fractures, and classic hyperextension injuries; there was only 1 study that involved patients with pure hyperextension injuries. We then segregated the evidence into 3 categories: 1) those that primarily compared surgery to conservative management; 2 ) those that addressed the timing of surgery; and 3) those that identified possible prognostic factors other than timing of surgical intervention.

Regarding conservative versus surgical management of TCCS, we identified 4 retrospective studies (Table 1). Three of these studies showed the superiority and safety of surgical intervention compared with conservative management. One study showed no difference in outcome; however, baseline characteristics between both groups were different as the surgically treated group had skeletal and discoligamentous injuries and the conservatively treated group suffered hyperextension injuries.

In regards to timing of surgery (early vs late), 6 studies were identified, all of which were retrospective in nature. The definition of early versus late varied from study to study (Table 2). Only 1 study (Yamazaki et al.) demonstrated improved outcome of early surgical intervention. ${ }^{26}$ In this study, early surgery was defined as within 2 weeks of the injury. The study by Guest et al. demonstrated superiority of early surgery (within 24 hours) in patients suffering from TCCS due to fractures..$^{12}$ Early surgery did not affect outcome in patients suffering from TCCS due to spondylosis. The other 3 studies did not demonstrate a significant difference between early surgical interventions (within 24-48 hours) and late intervention.

Six studies primarily investigated prognostic factors that would affect outcome in patients with TCCS (Table 3). Five of these studies identified older age as adversely affecting outcome; ${ }^{10,11,16,19,25} 1$ study did not support this claim..$^{13}$ Two studies found neurological state at the time of admission would affect outcome, with better outcomes resulting in patients presenting with better neurological examination results. ${ }^{11,25}$ One study found neurological state at the time of rehabilitation affected patient outcomes. ${ }^{13}$ Two studies showed the absence of spasticity correlated with better outcomes. ${ }^{11,25}$ One study showed formal education correlated with better outcomes. ${ }^{11}$ Lastly, 1 study demonstrated abnormal MR signal intensity correlated with worse outcomes. ${ }^{13}$

\section{Conclusions}

No Class I or II evidence was available to determine the efficacy of surgery, timing of surgery, or prognostic factors in patients managed for TCCS. We compiled 3 evidentiary tables summarizing 16 retrospective studies. Regarding surgical intervention compared with conservative therapy, there is Class III evidence to support the superiority of surgical intervention. In regard to timing of surgery, most Class III evidence demonstrated no difference in early versus late surgical intervention. Most Class III studies agreed that older age, especially older than 60 or 70 years, correlated with worse outcomes. There is a need to perform well-controlled prospective studies and randomized prospective trials to further investigate surgery versus conservative treatment as well as the timing of surgery in patients suffering from TCCS.

\section{Disclosure}

Dr. Fessler was the principal investigator of a research grant awarded to the Northwestern University Feinberg School of Medicine by Medtronic PS Medical for the development of minimally invasive spine surgical procedures. He is a member of the Scientific Advisory Board for Lanx, Inc. He also receives royalties from DePuy, Stryker, and Medtronic, but not related to minimally invasive techniques and technologies.

Author contributions to the study and manuscript preparation include the following. Conception and design: Fessler, Dahdaleh, Smith. Acquisition of data: Dahdaleh, Lawton, Nixon, Oh. Analysis and interpretation of data: Dahdaleh, Lawton, Oh. Drafting the article: Dahdaleh, Lawton, El Ahmadieh, Nixon, El Tecle, Oh. Critically revising the article: Fessler, Dahdaleh, Lawton, El Ahmadieh, Nixon, El Tecle. Reviewed submitted version of manuscript: Fessler, Dahdaleh, Lawton, Nixon, El Tecle, Smith. Approved the final version of the manuscript on behalf of all authors: Fessler. Study supervision: Fessler, Dahdaleh.

\section{References}

1. Aarabi B, Alexander M, Mirvis SE, Shanmuganathan K, Chesler D, Maulucci C, et al: Predictors of outcome in acute traumatic central cord syndrome due to spinal stenosis. Clinical article. J Neurosurg Spine 14:122-130, 2011

2. Aarabi B, Koltz M, Ibrahimi D: Hyperextension cervical spine injuries and traumatic central cord syndrome. Neurosurg Focus 25(5):E9, 2008

3. Aito S, D'Andrea M, Werhagen L, Farsetti L, Cappelli S, Bandini B, et al: Neurological and functional outcome in traumatic central cord syndrome. Spinal Cord 45:292-297, 2007

4. Anderson DG, Sayadipour A, Limthongkul W, Martin ND, Vaccaro A, Harrop JS: Traumatic central cord syndrome: neurologic recovery after surgical management. Am J Orthop 41:E104-E108, 2012

5. Bose B, Northrup BE, Osterholm JL, Cotler JM, DiTunno JF: Reanalysis of central cervical cord injury management. Neurosurgery 15:367-372, 1984

6. Chen L, Yang H, Yang T, Xu Y, Bao Z, Tang T: Effectiveness of surgical treatment for traumatic central cord syndrome. Clinical article. J Neurosurg Spine 10:3-8, 2009

7. Chen TY, Dickman CA, Eleraky M, Sonntag VKH: The role of decompression for acute incomplete cervical spinal cord injury in cervical spondylosis. Spine (Phila Pa 1976) 23:23982403, 1998

8. Chen TY, Lee ST, Lui TN, Wong CW, Yeh YS, Tzaan WC, et al: Efficacy of surgical treatment in traumatic central cord syndrome. Surg Neurol 48:435-441, 1997

9. Dai L, Jia L: Central cord injury complicating acute cervical disc herniation in trauma. Spine (Phila Pa 1976) 25:331-336, 2000

10. Dai LY: Acute central cervical cord injury: the effect of age upon prognosis. Injury 32:195-199, 2001

11. Dvorak MF, Fisher CG, Hoekema J, Boyd M, Noonan V, Wing PC, et al: Factors predicting motor recovery and functional outcome after traumatic central cord syndrome: a long-term follow-up. Spine (Phila Pa 1976) 30:2303-2311, 2005

12. Guest J, Eleraky MA, Apostolides PJ, Dickman CA, Sonntag VKH: Traumatic central cord syndrome: results of surgical management. J Neurosurg 97 (1 Suppl):25-32, 2002

13. Hohl JB, Lee JY, Horton JA, Rihn JA: A novel classification system for traumatic central cord syndrome: the central cord injury scale (CCIS). Spine (Phila Pa 1976) 35:E238-E243, 2010 


\section{Central cord syndrome}

14. Jimenez O, Marcillo A, Levi AD: A histopathological analysis of the human cervical spinal cord in patients with acute traumatic central cord syndrome. Spinal Cord 38:532-537, 2000

15. Koyanagi I, Iwasaki Y, Hida K, Imamura H, Fujimoto S, Akino M: Acute cervical cord injury associated with ossification of the posterior longitudinal ligament. Neurosurgery 53:887-892, 2003

16. Lenehan B, Street J, O'Toole P, Siddiqui A, Poynton A: Central cord syndrome in Ireland: the effect of age on clinical outcome. Eur Spine J 18:1458-1463, 2009

17. Maroon JC, Abla AA, Wilberger JI, Bailes JE, Sternau LL: Central cord syndrome. Clin Neurosurg 37:612-621, 1991

18. Matz PG, Anderson PA, Kaiser MG, Holly LT, Groff MW, Heary RF, et al: Introduction and methodology: guidelines for the surgical management of cervical degenerative disease. J Neurosurg Spine 11:101-103, 2009

19. Newey ML, Sen PK, Fraser RD: The long-term outcome after central cord syndrome: a study of the natural history. J Bone Joint Surg Br 82:851-855, 2000

20. Nowak DD, Lee JK, Gelb DE, Poelstra KA, Ludwig SC: Central cord syndrome. J Am Acad Orthop Surg 17:756-765, 2009

21. Quencer RM, Bunge RP, Egnor M, Green BA, Puckett W, Naidich TP, et al: Acute traumatic central cord syndrome: MRIpathological correlations. Neuroradiology 34:85-94, 1992

22. Schneider RC, Cherry G, Pantek H: The syndrome of acute central cervical spinal cord injury; with special reference to the mechanisms involved in hyperextension injuries of cervical spine. J Neurosurg 11:546-577, 1954

23. Smith ZA, Buchanan CC, Raphael D, Khoo LT: Ossification of the posterior longitudinal ligament: pathogenesis, management, and current surgical approaches. A review. Neurosurg Focus 30(3):E10, 2011

24. Stevens EA, Marsh R, Wilson JA, Sweasey TA, Branch CL Jr, Powers AK: A review of surgical intervention in the setting of traumatic central cord syndrome. Spine J 10:874-880, 2010

25. Tow AM, Kong KH: Central cord syndrome: functional outcome after rehabilitation. Spinal Cord 36:156-160, 1998

26. Yamazaki T, Yanaka K, Fujita K, Kamezaki T, Uemura K, Nose T: Traumatic central cord syndrome: analysis of factors affecting the outcome. Surg Neurol 63:95-100, 2005

Manuscript submitted March 12, 2013.

Accepted March 25, 2013.

Please include this information when citing this paper: DOI: 10.3171/2013.3.FOCUS13101.

Address correspondence to: Richard G. Fessler, M.D., Ph.D., Department of Neurological Surgery, Northwestern University Feinberg School of Medicine, 676 N. St. Clair St., Suite 2210, Chicago, IL 60611. email: rfessler@nmff.org. 